\title{
ON COMMUTATORS IN A SIMPLE LIE ALGEBRA ${ }^{1}$
}

\section{GORDON BROWN}

1. Introduction. K. Shoda [3] has shown that any $n \times n$ matrix of trace zero over a field of characteristic zero is expressible as an additive commutator. A. Albert and B. Muckenhoupt [1] have extended this result to fields of all characteristics. Rephrasing their result, it is easily seen that every element of a Lie algebra of type $A_{n}$ can be expressed as a Lie product. It seems natural to ask whether a similar assertion is valid for a wider class of Lie algebras.

The purpose of this paper is to employ a generalization of Shoda's method to show that any element of a classical Lie alsebra (defined by R. Steinberg [4]) can be expressed as a Lie product provided only that a certain restrictive assumption on the cardinality of the field $K$ over which the algebra is defined is satisfied. This restriction is made in this paper for the sole purpose of permitting a relatively simple method to be applied to prove the main theorem.

2. The notation and method of proof. The classical simple Lie algebras will be denoted by $L=L^{*} / Z$ where $Z$ is the center of $L^{*}$. $L^{*}$ will also be called classical. $K$ will denote the base field. $x y$ is the Lie product of $x$ and $y, H$ a standard Cartan subalgebra, $\phi$ runs through the nonzero roots of $L^{*}$ relative to $H L^{*}=H+E$, a vector space direct sum, where $E=\sum_{\phi} L_{\phi}$. Each $L_{\phi}$ is one-dimensional, and $H$ is spanned by $h_{i}=h_{\phi_{i}}(i=1, \cdots, n)$, where $\phi_{i}\left(h_{i}\right)=2, h_{i}=e_{\phi_{i}} e_{-\phi_{i}}$ for some $e_{\phi_{i}} \in L_{\phi_{i}}, e_{-\phi_{i}} \in L_{-\phi_{i}}$, the $\phi_{i}$ being a fundamental system of simple roots for $L^{*}$ relative to $H$. If $\phi$ and $\psi$ are roots, $\phi \neq-\psi$, then $L_{\phi} L_{\psi}=L_{\phi+\psi}$ if $\phi+\psi$ is a root, and 0 otherwise. $l$ and $l^{\prime}$ will be said to be conjugates of one another if $l^{\prime}$ is the image of $l$ under an automorphism of $L^{*}$. The difference $r-q$, where $\psi-r \chi$ and $\psi+q \chi$ are roots of $L^{*}$, but $\psi-(r+1) \chi$ and $\psi+(q+1) \chi$ are not roots, is denoted by $A_{\chi \psi}$, and $A_{\phi_{i} \phi_{j}}$ is abbreviated $A_{i j}$. The algebras of types $A_{1}, B_{n}$, $C_{n}, F_{4}$, and $G_{2}$ are not included in the list of classical Lie algebras if $K$ is of characteristic $p=2 . G_{2}$ is also excluded if $p=3$. The excluded algebras either are not simple or are isomorphic to other classical

Presented to the Society, April 14, 1962; received by the editors April 17, 1962 and, in revised form, August 17, 1962.

1 This paper is a portion of the author's doctoral dissertation. The author would like to express his sincere appreciation to Professor I. N. Herstein for proposing this problem to him and for his helpful suggestions during its preparation. Written with the support of Grant DA-ARO (D)-31-124-G86. 
algebras. Because of these exclusions, if $\chi \neq \pm \psi, A_{x \psi} \equiv 0(\bmod p)$ if and only if $A_{x \psi}=0$.

The aforementioned proof by Shoda was based on two lemmas, namely that any matrix all of whose diagonal elements are zero is expressible as a commutator, and secondly that any matrix of trace zero is similar to a matrix all of whose diagonal elements are zero. Such a matrix is an element of a Lie algebra of type $A_{n}$ belonging to the subspace $E$. We shall use this fact to rephrase Shoda's lemmas in the terminology of Lie algebra theory as follows:

Lemma I. There exists an $h \in H$ such that $\{h l \mid l \in L\}=E$.

Lemma II. Every element of $L$ has a conjugate in $E$.

For any algebra $L$ for which Lemma I and Lemma II are valid, we can prove

THEOREM A. Every element $s$ of $L$ can be expressed as a Lie product.

Proof. There exists an automorphism $g$ of $L$ such that $g(s)=h l$. Hence $s=g^{-1}(h) g^{-1}(l)$.

We shall prove that Lemma $I$ is valid for all classical algebras provided that the cardinality of $K$ exceeds $c$ where $c$ is $2 n-1$ for $A_{n}$, $4 n-5$ for $B_{n}$ and $C_{n}, 4 n-7$ for $D_{n}, 5$ for $G_{2}, 15$ for $F_{4}, 21$ for $E_{6}, 33$ for $E_{7}$, and 57 for $E_{8}$.

Lemma II will be proved valid for all classical Lie algebras.

Theorem $\mathrm{A}$ is therefore valid for all classical algebras for which Lemma $I$ is valid.

Let $L=L_{1} \oplus \cdots \oplus L_{n}$. Suppose that Theorem $\mathrm{A}$ is valid for $L_{i}$ for all $i$. Then $s \in L$ can be written $s=s_{1}+\cdots+s_{n}=x_{1} y_{1}+\cdots+x_{n} y_{n}$ $=\left(\sum_{i=1}^{n} x_{i}\right)\left(\sum_{i=1}^{n} y_{i}\right)$, and Theorem $\mathrm{A}$ is also valid for $L$.

3. Proof of Lemma I. Consider an element $a=\sum_{\phi} m_{\phi} e_{\phi}$. It is necessary to find an $h \in H$ such that $h\left(n_{\phi} e_{\phi}\right)=m_{\phi} e_{\phi}$ can be solved for $n_{\phi}$ for all $\phi$. Let $h=\sum_{i=1}^{n} q_{i} h_{i}$. Then $h\left(n_{\phi} e_{\phi}\right)=\left(\sum_{i} q_{i} \phi\left(h_{i}\right)\right) n_{\phi} e_{\phi}$. Therefore $h\left(n_{\phi} e_{\phi}\right)=m_{\phi} e_{\phi}$ can be solved for $n_{\phi}$ if $\sum_{i} q_{i} \phi\left(h_{i}\right) \neq 0$. Let $\sum_{i} \phi\left(h_{i}\right) x_{i}$ $=g_{\phi}\left(x_{1}, \cdots, x_{n}\right)$, a polynomial in $n$ variables. Since there exists an $h_{i}$ such that $\phi\left(h_{i}\right) \neq 0, g_{\phi}$ is not identically zero. Since $g_{\phi}=-g_{-\phi}$, we note that if $h\left(q_{1}, \cdots, q_{n}\right) \neq 0$ where $h=\prod_{\phi} g_{\phi}$, then $g_{\phi}\left(q_{1}, \cdots, q_{n}\right)$ $\neq 0$ for all $\phi$. Such $q_{i}$ exist provided only that the cardinality of $K$ exceeds the degree of $h\left(x_{1}, \cdots, x_{n}\right)$ in $x_{i}$ for all $i$. This degree can be calculated and found to be $c$, the number defined above. The validity of Lemma I for $L^{*}$ implies its validity for $L$. Therefore, with the exceptions stated above, Lemma $\mathrm{I}$ is valid for all classical Lie algebras. 


\section{Proof of Lemma II.}

LEMMA 4.1. If $a=\sum_{i=1}^{n} m_{i} h_{i}+\sum_{\phi} m_{\phi} e_{\phi} \notin Z$, then a has a conjugate $a^{\prime}=\sum m_{i}^{\prime} h_{i}+\sum m_{\phi}^{\prime} e_{\phi}$ such that $m_{j}^{\prime}=0$ for some $j$.

Proof. We let $x_{\phi}(y)$ be the automorphism of $L^{*}$ having the same effect as $\exp \left(\operatorname{ad} y e_{\phi}\right)$ on each generator, except that $x_{\phi}(y) e_{-\phi}=e_{-\phi}$ $+y h_{\phi}+y^{2} e_{\phi}$ if $K$ has characteristic 2 .

Suppose that there exists a root $\psi$ such that $m_{\psi} \neq 0$. Let $a^{\prime}$ $=\sum_{i=1}^{n} m_{i}^{\prime} h_{i}+\sum_{\phi} m_{\phi}^{\prime} e_{\phi}=x_{-\psi}(y) a$. Then

$$
m_{j}^{\prime}=m_{j}+k_{j} \frac{A_{\psi \phi_{j}}}{A_{\phi_{j} \psi}} m_{\psi} y,
$$

where $\psi=\sum_{i=1}^{n} k_{i} \phi_{i}$. It is easily observed that $k_{j}\left(A_{\psi \phi_{j}} / A_{\phi_{j} \psi}\right)$ $\equiv 0(\bmod p)$ does not hold for every $j$. Therefore there is a $j$ such that $m_{j}^{\prime}=0$ can be solved for $y$, and consequently Lemma 4.1 is valid if $a \notin H$.

Now suppose $m_{\phi}=0$ for all $\phi \neq 0$, i.e., $a \in H$. If it can be shown that $a$ is conjugate to an element $a^{\prime} \notin H$, then the lemma will follow by the transitivity of the conjugacy relation. Since $x_{\phi}(1) a$ is $a^{\prime}=\sum m_{i}^{\prime} h_{i}$ $+\sum m_{\phi}^{\prime} e_{\phi}$ where $m_{\phi}^{\prime} e_{\phi}=\left(\sum_{i=1}^{n} m_{i} h_{i}\right) e_{\phi}=a e_{\phi}, m_{\phi}^{\prime}=0$ for all $\phi$ only if $a e_{\phi}=0$ for all $\phi$. Since $a \in H$, and $H$ is abelian, $a h=0$ for $h \in H$. Thus the Lie product of $a$ with any generator would be zero, implying $a \in Z$, thus contradicting the hypothesis of the lemma.

LEMma 4.2. Let $S$ be an indecomposable subset of the fundamental system of roots for a classical Lie algebra $L^{*}$. Then the subalgebra $L_{S}^{*}$ generated by $\left\{e_{\phi_{i}}, e_{-\phi_{i}}\right\}$ for $\phi_{i} \in S$ is a classical Lie algebra unless $K$ is of characteristic 2 , and $S$ consists of only one root.

Proof. Let $L$ be a complex simple Lie algebra with Cartan subalgebra $H$ and fundamental system of roots $\phi_{1}, \cdots, \phi_{n}$. Let $S$ be an indecomposable subset of this fundamental system. Let $L_{S}$ be the subalgebra of $L$ generated by $\left\{e_{\phi_{i}}, e_{-\phi_{i}}\right\}$ for $\phi_{i} \in S$. Let $R$ be the linear transformation from the complex vector space $V_{S}$ spanned by $\phi_{i} \in S$ into the space of linear functionals on $H_{S}=H \cap L_{S}$, the space spanned by $\left\{h_{i}\right\}$ where $\phi_{i} \in S$, defined by $R(\lambda)=\lambda \mid H_{S}$. If $\lambda=\sum c_{i} \phi_{i}$ is in the kernel of $R$, then $\sum c_{i} \phi_{i}\left(h_{j}\right)=0$ for all $j$ such that $\phi_{j} \in S$. Since the matrix $\left(\phi_{i}\left(h_{j}\right)\right)=\left(A_{i j}\right)$ for $i, j$ such that $\phi_{i}, \phi_{j} \in S$ is a principal submatrix of the Cartan matrix of $L$, it is nonsingular by [2], and so $\lambda$ must be zero, and $R$ is one-to-one. Thus if the root $\psi$ is in $V_{S}$, there is an $h \in H_{S}$ such that $h e_{\psi}=\psi(h) e_{\psi} \neq 0$, and so $H_{S}=\left\{l \in L_{S}\right.$ : $l h=0$ for all $\left.h \in H_{S}\right\}$, i.e. $H_{S}$ is a Cartan subalgebra of $L_{S}$. Clearly, $\psi \mid H_{S}$ is a root of $H_{S}$ in $L_{S}$, and, conversely, every root of $H_{S}$ in $L_{S}$ 
is the restriction to $H_{S}$ of a root in $V_{S}$ since the elements $e_{\psi}, \psi$ a root in $V_{S}$, form a basis for $E_{S}=E \cap L_{S}$, and $L_{S}=H_{S}+E_{S}$. Since $R(\psi-\chi)$ is a root of $H_{S}$ in $L_{S}$ if and only if $\psi-\chi \in V_{S}$ is a root of $H$ in $L$, and since $R$ preserves linear independence, $\left\{\phi_{i} \mid H_{S}: \phi_{i} \in S\right\}$ is a fundamental system of simple roots for $L_{s}$. Therefore since $S$ is indecomposable, $L_{S}$ is simple.

Let $L_{z}$ be the Lie subring of $L$ consisting of all integral linear combinations of the basis $\left\{e_{\phi}, h_{i}\right\}$. Then $\hat{L}=L_{z} \otimes_{z} K$ is a Lie algebra over the base field $K$ if $\left(l_{1} \otimes k_{1}\right)\left(l_{2} \otimes k_{2}\right)=\left(l_{1} l_{2} \otimes k_{1} k_{2}\right)$. A comparison of their multiplication tables, identifying $l \otimes k$ with $k l$, reveals that $\hat{L}$ is isomorphic to the classical algebra $L^{*}$ defined in $\$ 2$ except when the type of $L$ and the characteristic of $K$ are specifically excluded by that definition. Similarly, $\hat{L}_{S}=\left(L_{S}\right)_{z} \otimes_{z} K$ is classical, with the same exceptions, since $L_{S}$ is simple. However, it is easily observed that the only algebra among these nonclassical algebras which can be a subalgebra of a classical algebra is the algebra of type $A_{1}$ over a field of characteristic 2. This establishes the lemma.

Similarly, if $S$ is decomposable, $L_{S}^{*}$ is a direct sum of classical Lie algebras unless $K$ has characteristic 2 , and $S$ has a maximal indecomposable subset containing only one root.

In order to establish Lemma II, we first prove

\section{Lemma $\mathrm{II}^{\prime}$. Every noncentral element of $L^{*}$ has a conjugate in $E$.}

The proof of Lemma II' will proceed by induction on the number of simple roots. First we observe that if $K$ has characteristic $p>2$, then Lemma II' $^{\prime}$ is valid for $A_{1}$ by Lemma 4.1 .

If $p=2$, we establish Lemma II' for $A_{2}$ as follows: Let $i=1, j=2$ or $i=2, j=1$. Suppose $a=\sum_{1}^{2} m_{k} h_{k}+\sum_{\phi} m_{\phi} e_{\phi}$. Let $m_{i} \neq 0$. We may assume $m_{\phi_{j}} \neq 0$ or $m_{-\phi_{j}} \neq 0$ since if $m_{\phi_{j}}=m_{-\phi_{j}}=0, x_{\phi_{j}}(1) a=a^{\prime}=\sum m_{k}^{\prime} h_{k}$ $+\sum m_{\phi}^{\prime} e_{\phi}$ where $m_{\phi_{j}}^{\prime} \neq 0$ and $m_{i}^{\prime}=m_{i}$. An appropriate value of $y$ can be found for either $x_{\phi_{j}}(y) a$ or $x_{-\phi_{j}}(y) a$ to yield an element with $m_{j}^{\prime}$ $=m_{i}^{\prime}$. If $m_{\phi_{1}+\phi_{2}}=m_{-\phi_{1}-\phi_{2}}=0, x_{\phi_{1}+\phi_{2}}(1)$ will yield $a^{\prime}$ with $m_{\phi_{1}+\phi_{2}}^{\prime} \neq 0$ and $m_{k}^{\prime}=m_{k}$. Therefore suppose $a=\sum m_{k} h_{k}+\sum m_{\phi} e_{\phi}, m_{i}=m_{j}$, and $m_{\phi_{1}+\phi_{2}} \neq 0$ or $m_{-\phi_{1}-\phi_{2}} \neq 0$. Then for appropriate $y \in K, x_{\phi_{1}+\phi_{2}}(y)$ or $x_{-\phi_{1}-\phi_{2}}(y)$ applied to $a$ yields an element such that $m_{1}^{\prime}=m_{2}^{\prime}=0$. Hence Lemma II' is valid for $A_{2}$ over a field $K$ of characteristic 2 .

Assume $a \in L^{*}, a \notin Z$, and that the cardinality of the fundamental system of roots of $L^{*}$ is $n$. If $K$ has characteristic $p>2$, assume that Lemma $\mathrm{II}^{\prime}$ has been proved for all algebras $L^{*}$ with fundamental systems of cardinality $c<n$ where $L^{*}$ is classical or the direct sum of classical algebras. If $p=2$, assume that Lemma $\mathrm{II}^{\prime}$ has been proved for all algebras with fundamental systems of cardinality $c$ where 
$2 \leqq c<n$. Let $a^{\prime}=\sum m_{i} h_{i}+\sum m_{\phi} e_{\phi}$ be a conjugate of $a$ such that the number of $i$ such that $m_{i} \neq 0$ is minimal. We wish to show $a^{\prime} \in E$.

Let $S$ be the subset consisting of those roots $\phi_{j}$ of $L^{*}$ for which $m_{j} \neq 0$. It is possible to choose $a^{\prime}$ in such a way that $\sum m_{i} h_{i}$ is not in the center $Z_{S}$ of $L_{S}^{*}$. To show this, write $L_{S}^{*}=L_{S_{1}}^{*} \oplus \cdots \oplus L_{S_{k}}^{*}$. Suppose $\sum m_{i} h_{i} \in Z_{S}$. Let $\phi_{l} \notin S$, but $A_{k l} \neq 0$ for some $\phi_{k} \in S_{i}$, where $S_{i}$ consists of the roots $\phi_{i_{1}}, \cdots, \phi_{i_{r}}$. Since $A_{1}$ has no center if $p>2$, and $A_{1}$ is excluded from the list of classical Lie algebras if $p=2$, we have $r>1$. Unless $m_{\phi_{k}+\phi_{l}}=m_{-\phi_{k}-\phi_{l}}=0$, automorphisms $x_{\phi_{k}+\phi_{l}}(y)$ or $x_{-\phi_{k}-\phi_{l}}(y)$ for appropriate $y$ map $a^{\prime}$ into an element with $m_{k}^{\prime}=0, m_{l}^{\prime} \neq 0$, $m_{i}^{\prime}=m_{i}$ for $i \neq k, l$. If $m_{\phi_{k}+\phi_{l}}=m_{-\phi_{k}-\phi_{l}}=0$, the automorphism $x_{\phi_{k}+\phi_{l}}$ (1) maps $a^{\prime}$ into an element for which $m_{\phi_{k}+\phi_{l}} \neq 0$, thus satisfying the hypothesis of the preceding statement. $\left(\sum_{i_{1}}^{i_{r}} m_{i} h_{i}-m_{k} h_{k}\right) e_{\psi}=-m_{k} h_{k} e_{\psi}$ $=-m_{k} A_{\phi_{k} \psi} e_{\psi}$ for $\psi \in L_{S \sim \phi_{k}}^{*}, m_{k} \neq 0$ by assumption. $A_{\phi_{k} \psi} \neq 0(\bmod p)$. Therefore $\sum m_{i}^{\prime} h_{i}$ summed over $i$ such that $\phi_{i} \in S \sim \phi_{k}$ is not in $Z_{S \sim \phi_{k}}$.

If $S_{i}$ is a single root $\phi_{i}$ for all $i$, and $p=2$, then $L_{S_{i} \cup \phi_{k}}$ where $A_{i k} \neq 0$ is $A_{2}$, and it is shown above that any element in $A_{2}$ has a conjugate in $E \cap A_{2}$. Since $x_{\phi}(y)$ for $\phi \in A_{2}$ leaves $m_{l}^{\prime}=m_{l}$ where $\phi_{l} \notin A_{2}$, we have obtained a contradiction of the minimality assumption.

For all other situations we proceed as follows. Let $a^{\prime} \in L_{S}^{*}$. Then by induction on the cardinality of the fundamental system, $a^{\prime}$ is conjugate to an element in $E_{S}=E \cap L_{S}^{*}$. Hence $a^{\prime} \in E$.

If $a^{\prime} \notin L_{S}^{*}$, then $a^{\prime} \in L_{S}^{*}+B$ where $B=\sum L_{\phi}$, summed over $\phi$ such that $L_{\phi} \Phi L_{S}^{*}$. If $L_{\phi} \subseteq L_{S}^{*}$ and $L_{\psi} \Phi L_{S}^{*}$, then $x_{\phi}(y) e_{\psi} \in B$. Therefore $x_{\phi}(y) b \in B$ for all $b \in B$.

Now we have $a^{\prime}=a^{\prime \prime}+b, a^{\prime \prime} \in L_{S}^{*}, b \in B$. By a sequence of automorphisms $x_{\phi}(y)$ where $L_{\phi} \subseteq L_{S}^{*}, a^{\prime}$ can be transformed into an element in $E_{S}+B=E$. This completes the proof of Lemma $\mathrm{II}^{\prime}$.

Lemma II follows immediately from Lemma II', since any automorphism of $L^{*}$ induces an automorphism of $L$, and an element in the center of $L^{*}$ has the image zero in $L$.

\section{REFERENCES}

1. A. A. Albert and Benjamin Muckenhoupt, On matrices of trace zero, Michigan Math. J. 4 (1957), 1-3.

2. W. H. Mills and G. B. Seligman, Lie algebras of classical type, J. Math. Mech. 6 (1957), 519-548.

3. K. Shoda, Einige Sätze über Matrizen, Japan J. Math. 13 (1937), 361-365.

4. Robert Steinberg, Automorphisms of classical Lie algebras, Pacific J. Math. 11 (1961), 1119-1129.

Cornell University 